\title{
Identification of behavioral and metabolic factors predicting adiposity sensitivity to both high fat and high carbohydrate diets in rats
}

\author{
Patrick Christian Even*, Nachiket A. Nadkarni, Catherine Chaumontet, Dalila Azzout-Marniche, \\ Gilles Fromentin and Daniel Tomé
}

UMR914 Nutrition Physiology and Ingestive Behavior, AgroParisTech, INRA, Paris, France

\section{Edited by:}

Jean-Pierre Montani, University of

Fribourg, Switzerland

Reviewed by:

Jean-Pierre Montani, University of

Fribourg, Switzerland

Catalina Pico, University of the

Balearic Islands, Spain

Gretha Boersma, Johns Hopkins

University, USA

*Correspondence:

Patrick Christian Even, UMR914

Nutrition Physiology and Ingestive

Behavior, AgroParisTech, INRA,

F-75005 Paris, France.

e-mail: even@agroparistech.fr
Individuals exhibit a great variation in their body weight (BW) gain response to a high fat diet. Identification of predictive factors would enable better directed intervention toward susceptible individuals to treat obesity, and uncover potential mechanisms for treatment targeting. We set out to identify predictive behavioral and metabolic factors in an outbred rat model. 12 rats were analyzed in metabolic cages for a period of 5 days during both high carbohydrate diet (HCD), and transition to a high fat diet (HFD). After a recovery period, rats were given a HFD for 6 days to identify those resistant or sensitive to it according to BW gain. Rats were dissected at the end of the study to analyze body composition. This showed that small differences in final BW hid large variations in adiposity, allowing separation of rats into a second classification (final adiposity). Since these rats had been fed a HCD during most of their life, under which most of the adiposity presumably evolved, we considered this carbohydrate-sensitivity or -resistance. Meal size and meal number were found to be good predictors of sensitivity to a HFD, intensity of motor activity and ingestion speed good predictors of sensitivity to a HCD. Rats that were sensitive to the HCD could be resistant to the HFD and vice versa. This points to four types of individuals (carbohydrate/fat resistant/sensitive) though our sample size inhibited deeper investigation of this. This contributes to the idea that to be "obesity prone" does not necessarily need a HFD, it can also happen under a HCD, and be a hidden adiposity change with stable BW.

Keywords: obesity prone, obesity resistant, rat, food intake, motor activity, energy metabolism, high fat diet/low fat diet, indirect calorimetry

\section{INTRODUCTION}

Stability of body weight (BW) and body composition requires that over time, energy expenditure equals caloric intake (CI), in other words that energy nutrient oxidation equals intake in order to achieve nutrient balance. There is however a great inter-individual variability in human as well as in many animal populations in the capacity to achieve this (Chang et al., 1990; Neel, 1999; Speakman, 2007; Prentice et al., 2008; Stoger, 2008).

Because of extensive metabolic capacities to store fat in adipose tissue, fat balance is usually difficult to achieve more particularly in some fat-sensitive (FS; FR being resistant) subjects for whom high fat diets (HFD) promote obesity (Flatt, 1987, 1988). Moreover, as glucose is the main precursor for lipogenesis, some sensitive subjects could efficiently convert glucose to fat (subsequently stored in adipose tissue) thus also leading to difficulty to achieve energy balance, whereas other less sensitive subjects could more readily adjust carbohydrate oxidation to carbohydrate

Abbreviations: BW, body weight; CI, caloric intake; CR, carbohydrate resistant; CS, carbohydrate sensitive; EE, energy expenditure; FI, food intake; FR, fat resistant; FS, fat sensitive; HCD, high carbohydrate diet; HFD, high fat diet; HPD, high protein diet; IMI, inter meal interval; MS, meal size; RQ, respiratory quotient; SPA, spontaneous physical activity; TEE, total energy expenditure. intake. Accordingly, high carbohydrate diets (HCD) could also promote obesity in carbohydrate-sensitive (CS; CR being resistant) subjects. In contrast, despite the fact that the underlying mechanisms are not clarified, it is clearly established that high protein diets (HPD) do not promote obesity (Westerterp-Plantenga et al., 2001; Lacroix et al., 2004; Pichon et al., 2006; Layman et al., 2009).

The mechanisms potentially responsible for the sensitivity to fat have been extensively investigated using genetically bred FS rats that offer a well-defined population in the study of metabolic and behavioral characteristics related to sustained sensitivity or resistance to fat (Levin et al., 1997). Another approach is also to select subjects in a population of outbred rats (Chang et al., 1990). Selection is based on the level of sensitivity to HFD and additionally metabolic and/or behavioral component(s) that participate in this sensitivity. Many differences have been reported in separate studies in FS rats such as abnormal sympathetic nervous activity, increased release of norepinephrine after a glucose load, impaired growth hormone secretion, and suppression of glucose appearance by insulin (Ji and Friedman, 2003). It is not clear whether all these differences are present simultaneously in all FS rats or more probably reflect various defects acting alone or in conjunction to favor body fat gain, thus suggesting a complex distribution of rats according to their sensitivity to body fat accretion. 
This study hypothesized that differences can be observed not only under HFD (FS vs. FR) but also under HCD (CS vs. CS). Under these conditions, this work aims to characterize rats according to their relative resistance to fat gain under HFD or HCD and to investigate if various metabolic and/or behavioral parameters easily accessible before the rats are overweight and when fed a standard HCD can be good predictors of the specific sensitivity of individuals to HFD or HCD. For this purpose energy expenditure and behavioral differences were determined between FS and FR rats fed a low fat diet (HCD) and submitted to a short period of HFD. The experiment aimed to measure total energy expenditure (TEE), CI, and spontaneous physical activity (SPA) under free-feeding conditions during HCD and transition to HFD.

\section{EXPERIMENTAL METHODS \\ ANIMALS, HOUSING, AND DIETS}

The experimental protocol was approved by the French National Animal Care Committee. Male Wistar rats (Harlan Laboratories) weighed around $225 \mathrm{~g}$ (7 weeks) at their arrival in the laboratory. With the goal of keeping all the rats in the study and thus to avoid sacrificing many animals, the breeder was asked to select from its colony of 7-week-old rats the six lightest and the six heaviest in order to get rats with a large phenotypic heterogeneity. The composition of the diets is presented in Table 1. Animal facility conditions were a 12:12 Light-Dark cycle and $22^{\circ} \mathrm{C}$.

\section{DESIGN}

This is outlined in Figure 1. The rats were weighed 2 days after arrival in the laboratory. After the selection performed by the breeder, the range of BWs of the rats was large (206-258 g) but the mean weights of the light and heavy rats not substantially different

Table 1 | Nutrient composition of the high carbohydrate (HCD) and high fat (HFD) diets.

\begin{tabular}{lcc}
\hline & HCD & HFD \\
\cline { 2 - 3 } & \multicolumn{2}{c}{ Amount (g) } \\
\hline Milk protein & 140.0 & 170.0 \\
Corn starch & 622.4 & 436.6 \\
Saccharose & 100.3 & 71.1 \\
Soybean oil & 40.0 & 225.0 \\
Mineral mix & 35.0 & 35.0 \\
Vitamin mix & 10.0 & 10.0 \\
Cellulose & 50.0 & 50.0 \\
Choline & 2.3 & 2.3 \\
Total & 1000 & 1000 \\
& & Energy (\%) \\
Milk protein & 14.7 & \\
Corn starch & 65.3 & 14.4 \\
Saccharose & 10.5 & 36.9 \\
Soybean oil & 9.4 & 6.0 \\
Total & 100 & 42.8 \\
Energy (kJ/g) & 15.95 & 100 \\
Food quotient & 0.946 & 0.847 \\
& &
\end{tabular}

$(219.8 \pm 5.1$ vs $233.5 \pm 4.6, P=0.07)$ with individuals overlapping between the two groups. After arrival and 5 days of adaptation to the animal facility, the 12 rats were split into three groups of 4 rats (the number of rats that could be studied simultaneously in the behavioral-metabolic device) and maintained under HCD in order to be studied in turn by group $(1,2$, and 3 weeks after arrival in the laboratory). During a 5-day period each of the three groups was characterized for TEE, food intake (FI), and SPA during transition from HCD to HFD. After completion of the 5-days of calorimetric measurements, the rats were allowed to recover for 10 days under HCD, and then fed the HFD for 6 days to allow selection from BW gain between FS and FR rats. Body composition was analyzed from dissection and weighing of the main organs and tissues just after the end of the 6-day HFD period. These 12 rats could readily be separated into FS and FR groups, each of six rats with a highly significant difference in their BW gain response to the HFD. This suggested that despite initial small differences in BW, the selection performed by the breeder helped in the creation of a group with a large heterogeneity. There was however no correlation between the initial weight of the rats and their weight gain during the HFD period.

\section{MEASUREMENT OF THE COMPONENTS OF ENERGY EXPENDITURE}

The goal was to obtain for each rat measures of FI pattern, SPA, TEE and respiratory quotient (RQ) during HCD, HFD and the transition between the two. Groups of four rats were housed at 18:00 in individual metabolic cages equipped with a weighed food cup (sensitivity better than $0.05 \mathrm{~g}$ ) and an activity platform placed below (sensitivity better than $1 \mathrm{~g}$ ). For gas analysis, the cages were multiplexed - all connected to the same gas analyzers. Thus $\mathrm{VO}_{2}$ and $\mathrm{VCO}_{2}$ were measured on each cage during $2 \mathrm{~min}$ every $10 \mathrm{~min}$ ( $2 \mathrm{~min}$ for each cage, plus $2 \mathrm{~min}$ on room air to correct values for room $\% \mathrm{O}_{2}$ and $\left.\% \mathrm{CO}_{2}\right)$. To reduce close to zero any energy expenditure for thermoregulation (non-shivering thermogenesis), temperature in the room was maintained at 25$26^{\circ} \mathrm{C}$ in order to maintain in the metabolic cage a temperature of $26-27^{\circ} \mathrm{C}$.

Day 1 in the metabolic device was used for habituation. $\mathrm{VO}_{2}-$ $\mathrm{VCO}_{2}$ and FI were measured during day 2 (HCD feeding) and during days 3-5 the rats were switched to the HFD. For each cage FI and SPA were measured in $5 \mathrm{~s}$ time bins on a separate computer. For analysis, data were pooled into $10 \mathrm{~min}$ bins and combined with the $\mathrm{VO}_{2}-\mathrm{VCO}_{2}$ data. Metabolic rate was computed from $\mathrm{VO}_{2}$ and $\mathrm{VCO}_{2}$ according to the Weir formula (Ferrannini, 1988; Even et al., 1994).

\section{ANALYSIS OF BODY COMPOSITION}

Rats were deeply anesthetized with an overdose of pentobarbital $(40 \mathrm{mg} / \mathrm{kg})$ then killed following exsanguination by blood sampling from the vena cava. The main tissues and organs (liver, spleen, kidneys, brain, heart, subcutaneous, retroperitoneal, inguinal, and mesenteric fat pads, skin) were dissected out, blotted dry, and weighed to the nearest $0.01 \mathrm{~g}$.

\section{STATISTICAL ANALYSIS}

Differences between groups (FR vs. FS and CR vs. CS) were assessed using Student's $t$-test. A difference was considered statistically significant at $P<0.025$ (Bonferroni correction) to take 


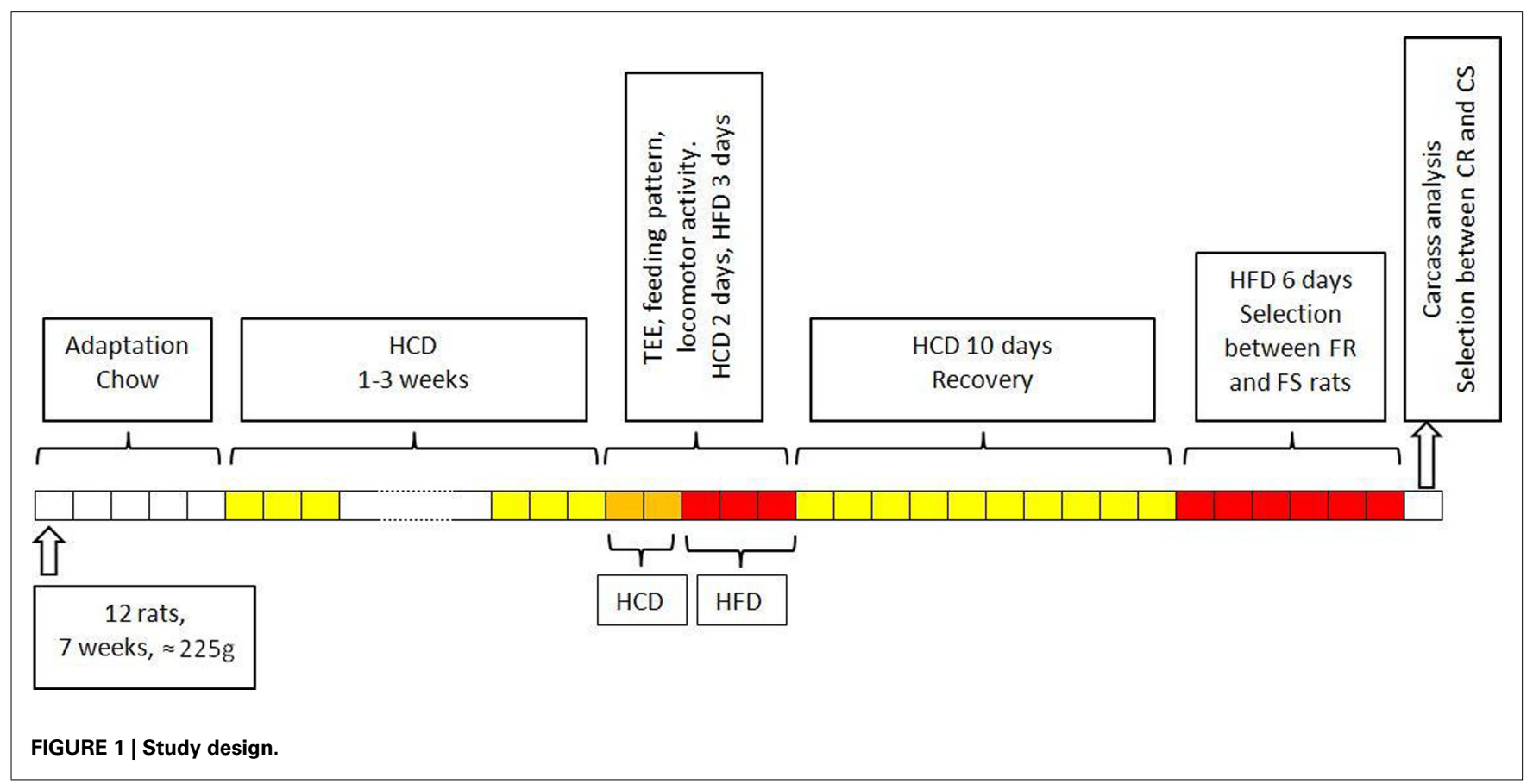

Table 2 | Body weight, composition, and weight gain of the FR and FS or CR and CS rats during the various periods (numbers of rats in each group in parentheses).

\begin{tabular}{|c|c|c|c|c|c|c|c|c|c|c|}
\hline & \multicolumn{5}{|c|}{ FR vs. FS } & \multicolumn{5}{|c|}{ CR vs. CS } \\
\hline & \multicolumn{2}{|c|}{$\mathrm{FR}(6)$} & \multicolumn{2}{|c|}{$\mathrm{FS}(6)$} & $\boldsymbol{P}$ & \multicolumn{2}{|c|}{$\operatorname{CR}(5)$} & \multicolumn{2}{|c|}{$\operatorname{cs}(7)$} & $P$ \\
\hline Body weight gain during HFD (6 days) & 21.67 & 2.02 & 31.15 & 1.80 & 0.006 & 27.42 & 4.10 & 25.69 & 1.86 & NS \\
\hline Final BW (g) & 353.85 & 8.58 & 354.70 & 20.60 & NS & 336.34 & 8.40 & 367.09 & 16.02 & NS \\
\hline Body fat (g) & 46.56 & 4.90 & 48.51 & 6.47 & NS & 36.12 & 4.19 & 55.68 & 3.52 & 0.006 \\
\hline Adiposity (\%) & 13.04 & 1.12 & 13.50 & 1.32 & NS & 10.68 & 1.07 & 15.12 & 0.49 & 0.010 \\
\hline
\end{tabular}

$S E$, standard error (of the mean). P, result of a two-tailed Student's t-test, d.f. =10. NS, not significant.

into account that the tests were repeated in the FR-FS and CR-CS study. Pearson product moment correlation analysis was also used to examine the relationship between the level of sensitivity to fat or carbohydrates and various metabolic and behavioral parameters that showed a trend toward significance between FR/FS or CR/CS rats.

\section{RESULTS}

CLASSIFICATION OF FS VS. FR AND CS VS. CR RATS ACCORDING TO BW GAIN AND BODY COMPOSITION

Body weight and composition data are shown in Table 2. The six FS rats were selected according to their larger daily BW gain during the period of HFD. Accordingly, BW gain during HF feeding was $44 \%$ larger in the FS than in the FR rats but no differences in body adiposity were observed between the two groups at the end of the study. However, within each group and therefore amongst all 12 rats combined, there was a great variation in adiposity. This allowed a second classification according to adiposity into five leaner rats (mean adiposity $10.68 \pm 1.07 \%$ ) and seven fattier rats (mean adiposity $15.12 \pm 0.49 \%$ ). We refer to the leaner rats as CR and to the fattier as CS since the adiposity differences were accumulated in rats fed under HCD during most of their life. In addition, because the CR and CS rats were approximately evenly distributed (five vs seven) into the FS and FR groups, we assumed that the 6-days of HFD affected similarly the body fat gain during this period in the CR and CS rats. Despite these large differences in body fat and body adiposity, final BWs of the CS and CR rats were not significantly different. Of the 31-g difference in BW, $20 \mathrm{~g}$ was accounted for by body fat $(+53 \%$ in the CS rats) while only $10 \mathrm{~g}$ was accounted for by LBM $(+3.7 \%$ in the CS rats). BW gain during HF feeding was also very similar in $\mathrm{CR}$ and CS rats. 


\section{COMPARISON OF THE COMPONENTS OF ENERGY EXPENDITURE FOR RATS CLASSIFIED AS FS VS. FR}

The meal pattern under HCD in rats classified as FS was characterized by significantly more numerous meals compared with FR during HCD but the difference decreased during HFD (Table 3 ). In parallel, inter-meal interval (IMI) and meal size tended to be lower which explains the absence of a significantly higher overall CI. No differences were observed between FR and FS rats for the components of SPA (Table 4). TEE was similar in FS and FR rats under $\mathrm{HCD}$, but decreased progressively in FS rats during HFD and became significantly lower than in FR rats after 3 days (Table 5). When expressed relative to HCD, TEE during HFD was significantly more decreased in FS rats after 2 and 3 days of HFD. In parallel, RQ tended to decrease more in FR rats, indicating a better adaptation to the increased fat content of the diet, and became significantly lower in FR than FS rats after 3 days of HFD. In summary, FS rats can be distinguished from FR rats from their larger meal number under HCD, and a larger decrease in TEE and maintenance of a higher RQ in response to HFD.

\section{COMPARISON OF THE COMPONENTS OF ENERGY EXPENDITURE FOR RATS CLASSIFIED AS CS VS. CR}

None of the parameters of FI showed significant differences between CR and CS rats during HCD as well as during HFD. In both groups, HFD increased CI. CS rats tended to spend more time active than $\mathrm{CR}$ rats during $\mathrm{HCD}(+23 \%, P=0.054$; Table 4$)$, but this tendency disappeared completely during HFD. In contrast, during HCD CS rats developed bursts of SPA that were of significantly lower intensity $(-29 \%, P=0.004$, ) than those recorded in the CR rats. This characteristic vanished progressively under HFD. No differences were observed between the two groups in TEE or RQ throughout the 4-days (Table 5). In summary, CS rats can be characterized primarily from the lower intensity of their bursts of SPA relative to CR rats during HCD.

\section{PREDICTION POTENTIAL OF THE METABOLIC AND BEHAVIORAL PARAMETERS FOR FS/FR AND CS/CR RATS}

According to the differences observed in the FS/FR and CS/CR rats, we re-analyzed the data starting from the potential predictive parameters to test if these parameters could indeed be good predictive factors.

\section{Predicting the FS/FR phenotype}

Fat-sensitive rats appeared to eat significantly more meals when fed the HCD than FR rats. Classification of the rats in accordance with meal number required that one rat that ate a large number of meals $(n=21)$ and was initially in the FR group had to be included in the group of potentially FS rats. This rat was lean (body fat $8.92 \%$ ), but was indeed the FR rat that exhibited the largest BW gain during the 6-days of HF feeding (25.5 g). Recalculation of BW gain and body composition of the two groups thus created according to meal frequency showed that they were indeed characterized by a very significant difference in weight gain during HF feeding (FS vs. FR; $29.00 \pm 1.28$ vs. $22.43 \pm 2.21 \mathrm{~g}, P=0.024$, d.f. $=10$ ).

Table 3 | Evolution of meal patterns in FS, FR and CR, CS rats in Design 2.

\begin{tabular}{|c|c|c|c|c|c|c|c|c|c|c|c|}
\hline & & \multicolumn{5}{|c|}{ FR vs. FS } & \multicolumn{5}{|c|}{ CR vs. CS } \\
\hline & & \multicolumn{2}{|c|}{ FR(6) } & \multicolumn{2}{|c|}{$\mathrm{FS}(6)$} & \multirow[t]{2}{*}{$\boldsymbol{P}$} & \multicolumn{2}{|c|}{ CR(5) } & \multicolumn{2}{|c|}{$\operatorname{cs}(7)$} & \multirow[t]{2}{*}{$P$} \\
\hline & & Mean & SE & Mean & SE & & Mean & SE & Mean & SE & \\
\hline \multirow[t]{4}{*}{ Caloric intake (kJ) } & $\mathrm{HCD}$ & 293.30 & 17.99 & 312.54 & 6.69 & NS & 293.72 & 22.59 & 309.20 & 5.86 & NS \\
\hline & HFD day 1 & 374.05 & 13.39 & 360.66 & 7.95 & NS & 361.50 & 10.46 & 371.54 & 11.72 & NS \\
\hline & HFD day 2 & 389.53 & 15.90 & 359.41 & 15.48 & NS & 360.66 & 23.01 & 384.09 & 11.30 & NS \\
\hline & HFD day 3 & 263.17 & 39.75 & 292.46 & 43.51 & NS & 221.75 & 10.04 & 317.57 & 43.10 & NS \\
\hline \multirow[t]{4}{*}{ Meal number } & $\mathrm{HCD}$ & 15.3 & 1.6 & 21.3 & 1.1 & 0.013 & 19.0 & 1.6 & 17.9 & 2.0 & NS \\
\hline & HFD day 1 & 15.2 & 1.2 & 20.8 & 1.7 & 0.023 & 17.8 & 1.6 & 18.1 & 2.0 & NS \\
\hline & HFD day 2 & 13.0 & 1.0 & 18.2 & 1.7 & NS & 15.0 & 1.3 & 16.0 & 1.9 & NS \\
\hline & HFD day 3 & 9.8 & 0.9 & 13.2 & 1.2 & NS & 10.8 & 1.0 & 12.0 & 1.3 & NS \\
\hline \multirow[t]{4}{*}{ IMI (min) } & $\mathrm{HCD}$ & 72.6 & 6.5 & 54.8 & 4.4 & NS & 58.5 & 5.2 & 67.4 & 6.9 & NS \\
\hline & HFD day 1 & 84.1 & 5.9 & 61.1 & 5.0 & 0.015 & 72.3 & 6.5 & 72.6 & 7.8 & NS \\
\hline & HFD day 2 & 87.2 & 8.1 & 73.4 & 6.9 & NS & 77.5 & 6.4 & 82.3 & 8.5 & NS \\
\hline & HFD day 3 & 103.9 & 6.9 & 92.1 & 6.4 & NS & 97.4 & 4.5 & 98.5 & 7.9 & NS \\
\hline \multirow[t]{4}{*}{ Meal size (kJ) } & $\mathrm{HCD}$ & 20.75 & 3.18 & 14.85 & 0.96 & NS & 16.19 & 2.47 & 19.00 & 2.64 & NS \\
\hline & HFD day 1 & 25.36 & 1.84 & 17.99 & 1.72 & 0.015 & 21.05 & 2.01 & 22.13 & 2.55 & NS \\
\hline & HFD day 2 & 30.84 & 2.68 & 21.00 & 2.80 & NS & 24.89 & 2.80 & 26.65 & 3.68 & NS \\
\hline & HFD day 3 & 26.78 & 3.01 & 24.02 & 5.48 & NS & 21.05 & 1.38 & 28.49 & 4.85 & NS \\
\hline \multirow[t]{4}{*}{ Speed (kJ/min) } & $\mathrm{HCD}$ & 2.50 & 0.27 & 2.49 & 0.21 & NS & 2.18 & 0.26 & 2.72 & 0.18 & NS \\
\hline & HFD day 1 & 3.90 & 0.30 & 5.40 & 1.03 & NS & 4.07 & 0.46 & 5.06 & 0.90 & NS \\
\hline & HFD day 2 & 4.23 & 0.63 & 4.48 & 0.21 & NS & 3.98 & 0.51 & 4.64 & 0.41 & NS \\
\hline & HFD day 3 & 4.10 & 0.88 & 3.89 & 0.59 & NS & 3.53 & 0.48 & 4.31 & 0.81 & NS \\
\hline
\end{tabular}

SE, standard error (of the mean). P, result of a two-tailed Student's t-test, d.f. $=10 . I M I$, inter-meal interval. NS, not significant. 
Table 4 | Components of spontaneous activity in FS, FR and CR, CS rats in Design 2 (numbers of rats in each group in parentheses).

\begin{tabular}{|c|c|c|c|c|c|c|c|c|c|c|c|}
\hline & \multirow[t]{2}{*}{ Whole day activity } & \multicolumn{5}{|c|}{ FR vs. FS } & \multicolumn{5}{|c|}{ CR vs. CS } \\
\hline & & \multicolumn{2}{|c|}{$\mathrm{FR}(6)$} & \multicolumn{2}{|c|}{$\mathrm{FS}(6)$} & $P$ & \multicolumn{2}{|c|}{ CR (5) } & \multicolumn{2}{|c|}{$\operatorname{cs}(7)$} & $P$ \\
\hline \multirow[t]{2}{*}{ Time active (min) } & HCD & 62.19 & 5.97 & 60.65 & 4.32 & NS & 54.119 & 1.655 & 66.635 & 5.176 & 0.054 \\
\hline & HFD day 1 & 64.57 & 4.43 & 75.92 & 4.18 & NS & 69.284 & 5.779 & 70.938 & 4.427 & NS \\
\hline \multirow[t]{4}{*}{ Mean act (U/100 g) } & $\mathrm{HCD}$ & 1.777 & 0.140 & 1.904 & 0.161 & NS & 2.024 & 0.147 & 1.710 & 0.129 & NS \\
\hline & HFD day 1 & 1.828 & 0.129 & 1.886 & 0.178 & NS & 2.089 & 0.157 & 1.692 & 0.109 & NS \\
\hline & HFD day 2 & 1.808 & 0.092 & 2.041 & 0.147 & NS & 2.119 & 0.098 & 1.785 & 0.115 & NS \\
\hline & HFD day 3 & 2.118 & 0.156 & 2.012 & 0.152 & NS & 2.301 & 0.054 & 1.897 & 0.148 & NS \\
\hline Intensity of bursts (U/100 g) & $\mathrm{HCD}$ & 2.962 & 0.326 & 3.206 & 0.328 & NS & 3.723 & 0.167 & 2.627 & 0.246 & 0.004 \\
\hline
\end{tabular}

Activity is measured by means of an activity platform in which force transducers record the work developed on the floor of the cage while the animal is active (see

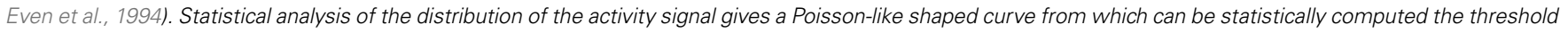

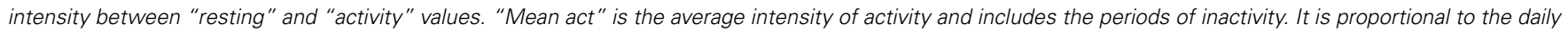
work expended with activity. "Intensity of bursts" is the average intensity of activity developed during the periods of activity. SE, standard error (of the mean). P, result of a two-tailed Student's t-test, d.f. $=10$.

Table 5 | Components of energy expenditure in FR and FS or CR and CS rats during low and high fat feeding in Design 2 (numbers of rats in each group in parentheses).

\begin{tabular}{|c|c|c|c|c|c|c|c|c|c|c|c|}
\hline & & \multicolumn{5}{|c|}{ FR vs. FS } & \multicolumn{5}{|c|}{ CR vs. CS } \\
\hline & & \multicolumn{2}{|c|}{$F R(6)$} & \multicolumn{2}{|c|}{$\mathrm{FS}(6)$} & \multirow[t]{2}{*}{$\boldsymbol{P}$} & \multicolumn{2}{|c|}{ CR(5) } & \multicolumn{2}{|c|}{$\operatorname{cs}(7)$} & \multirow[t]{2}{*}{$P$} \\
\hline & & Mean & SE & Mean & SE & & Mean & SE & Mean & SE & \\
\hline \multirow[t]{4}{*}{$E E(k J)$} & $\mathrm{HCD}$ & 232.30 & 2.76 & 242.96 & 6.74 & NS & 236.77 & 5.27 & 238.28 & 5.69 & NS \\
\hline & HFD day 1 & 219.95 & 5.27 & 230.87 & 5.73 & NS & 222.84 & 7.03 & 227.23 & 5.19 & NS \\
\hline & HFD day 2 & 230.62 & 3.31 & 228.45 & 6.65 & NS & 231.33 & 3.22 & 228.24 & 5.86 & NS \\
\hline & HFD day 3 & 230.54 & 2.59 & 219.79 & 8.79 & 0.01 & 227.07 & 5.69 & 223.80 & 7.20 & NS \\
\hline \multirow[t]{3}{*}{ dEE vs. HCD (\%) } & HFD day 1 & -22.51 & 5.31 & -20.63 & 3.18 & NS & -24.77 & 6.53 & -19.25 & 2.26 & NS \\
\hline & HFD day 2 & -2.93 & 5.82 & -24.98 & 2.97 & 0.01 & -8.87 & 9.41 & -17.57 & 4.23 & NS \\
\hline & HFD day 3 & -2.89 & 7.03 & -40.33 & 6.40 & 0.00 & -15.98 & 15.69 & -25.61 & 6.40 & NS \\
\hline \multirow[t]{4}{*}{$\mathrm{RQ}$} & $\mathrm{HCD}$ & 1.028 & 0.016 & 1.033 & 0.007 & NS & 1.018 & 0.019 & 1.040 & 0.006 & NS \\
\hline & HFD day 1 & 0.955 & 0.008 & 0.982 & 0.013 & NS & 0.965 & 0.015 & 0.971 & 0.010 & NS \\
\hline & HFD day 2 & 0.943 & 0.005 & 0.955 & 0.011 & NS & 0.946 & 0.011 & 0.951 & 0.008 & NS \\
\hline & HFD day 3 & 0.91 & 0.01 & 0.95 & 0.00 & 0.01 & 0.92 & 0.01 & 0.94 & 0.01 & NS \\
\hline \multirow[t]{3}{*}{ dRQ vs. HCD (\%) } & HFD day 1 & -7.08 & 0.85 & -4.96 & 0.87 & NS & -5.15 & 0.91 & -6.64 & 0.91 & NS \\
\hline & HFD day 2 & -8.25 & 1.21 & -7.56 & 0.54 & NS & -6.96 & 1.03 & -8.58 & 0.78 & NS \\
\hline & HFD day 3 & -11.29 & 1.87 & -8.04 & 0.44 & NS & -9.35 & 1.80 & -9.88 & 1.35 & NS \\
\hline
\end{tabular}

For a better adjustment of energy expenditure between rats, we avoided adjustment of energy expenditure based on whole body weight, and instead used LBM. This is because carcass analysis performed only 2 weeks after the calorimetric studies revealed that 2/3 of the differences in body weight were accounted for by differences in body fat. We thus assumed that body adiposity during the calorimetric studies was not very different from that measured from carcass analysis. The computed LBM value was used to normalize energy expenditure (EE) between rats. SE, standard error (of the mean). $P$, result of a two-tailed Student's t-test, $d . f .=10$.

Analysis of their metabolic and behavioral characteristics logically increased the differences under HCD (but also under HFD) in meal number $(21.5 \pm 1.1$ vs. $13.5 \pm 1.6, P=0.002$, d.f. $=10$ during
HCD), MS ( $13.9 \pm 1.17$ vs. $24.5 \pm 3.18 \mathrm{~kJ}, P=0.007$, d.f. $=10)$, and IMI $(53.5 \pm 4.3$ vs. $81.0 \pm 5.6 \mathrm{~min}, P=0.004$, d.f. $=10)$, which became significantly different. The decrease in RQ measured after 
3 days of HF feeding was still smaller in FS rats $(-7.374 \pm 0.552$ vs. $-13.042 \pm 2.157, P=0.02$, d.f. $=10)$. In contrast the decrease in TEE remained larger but was no more significant. As a result the numbers of meals eaten by a rat during HCD and/or the decrease in RQ in response to HF feeding are two parameters that discriminate FR and FS rats. Correlation analyses performed on these parameters showed that in 10 of the 12 rats the level of sensitivity to HFD was correlated to MN, IMI, MS, and the HFD-induced decrease in RQ (Figure 2). However, for all the correlations, the two same rats, the lowest and the highest BW gainers lay away from the regression lines suggesting that for these very resistant and sensitive individuals specific metabolic characteristics not shared by the other rats may be involved. If these two rats are taken into account only the correlation between $\mathrm{BW}$ gain and meal number remains significant $(P<0.02)$.

\section{Predicting the CR/CS phenotype}

The intensity of the bursts of activity was the parameter most significantly different between CR and CS rats. Classification of the rats according to the intensity of the bursts of activity required that one rat previously classified as CS that exhibited bursts of high intensity $(3.97 \mathrm{U} / 100 \mathrm{~g})$ had to be classified as potentially CR. This rat was the one with the second lowest adiposity level (13.59\%). Recalculation of body composition of the two groups following intensity of activity bursts confirmed large differences in body fat between the two groups $(37.48 \pm 3.68$ vs. $57.58 \pm 3.51 \mathrm{~g}, P=0.003)$, increased the differences in burstintensity $(3.764 \pm 0.143$ vs. $2.403 \pm 0.119 \mathrm{U} / 100 \mathrm{~g}, P=0.00003$, d.f. $=10)$, and made overall activity significantly different between CR and CS rats $(2.073 \pm 0.130$ vs. $1.608 \pm 0.095 \mathrm{U} / 100 \mathrm{~g}$,
$P=0.018$, d.f. $=10)$. In addition these differences under HCD remained significant during the 3-days of HFD. As a result, the intensity of bursts of activity and overall activity recorded in HCD fed rats are two parameters that discriminate CR and CS rats. Correlation analyses showed that a close quantitative relationship existed during HCD between the sensitivity to HCD and the intensity of the bursts of SPA. Mean SPA intensity and RQ were also potential predictors of CS, but were less accurate. No individuals appeared to diverge in the CR and CS rats (Figure 3). Interestingly, the level of carbohydrate sensitivity also correlated tightly with the speed of ingestion; however a large overlap between the speed of ingestion and the level of CS prevents speed of ingestion being a reliable predictive parameter (Figure 3 ).

\section{DISCUSSION}

First of all, it is important to discuss several limitations of this study. First, to avoid unnecessary sacrifice of animals, we assumed that a low number of animals could be used if an initial preselection of the rats by the breeder in a very large colony lead to initial important differences in their sensitivity to BW gain. This assumption seemed to work rather well because we were able to discriminate FR and FS rats that displayed highly significant differences in BW gain during HFD. In addition, the individuals ended the study with large differences in their adiposity (CR and CS rats). The fact remains however that the low number of animals reduces the statistical power available to discriminate between the various dietary CR/CS or FR/FS phenotypes. Additionally, because only four rats could be studied each week in the metabolic cages, sessions were staggered by 1-2 weeks, potentially contributing to variability in weight gain and body composition. We are rather
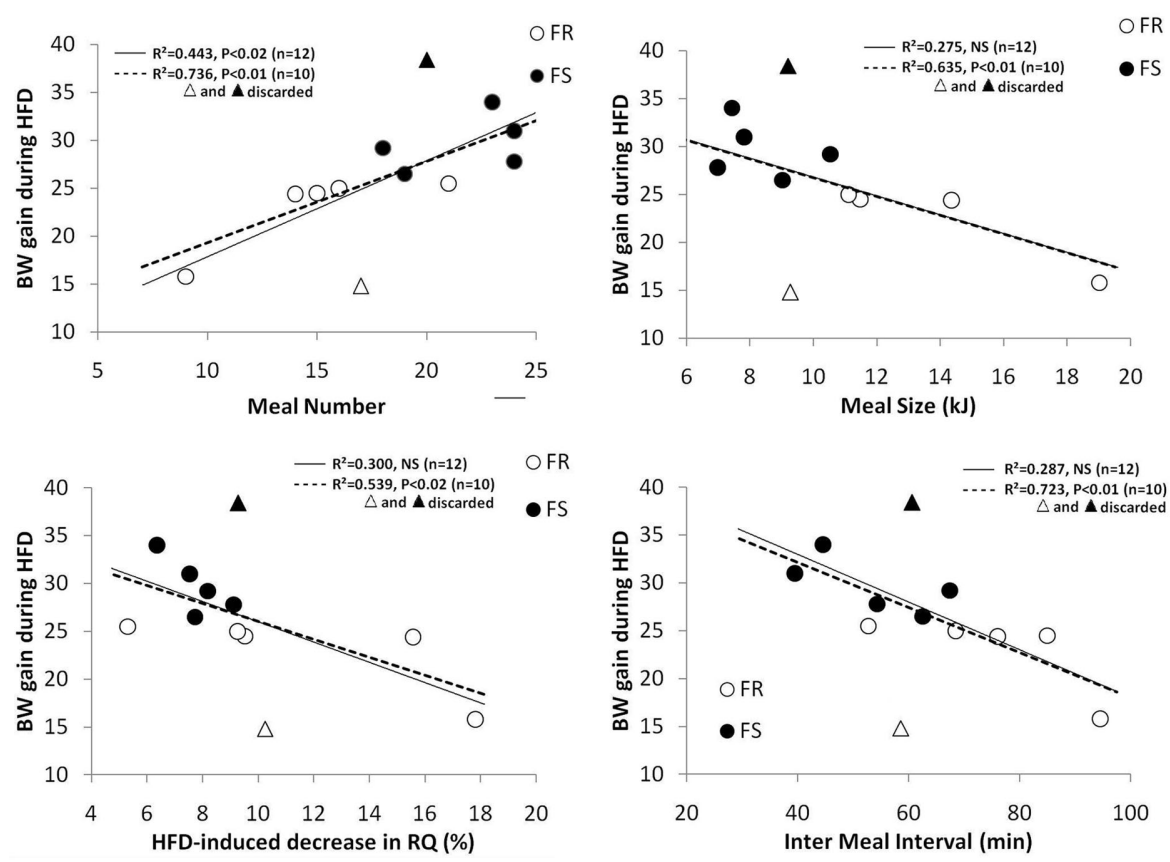

FIGURE 2 | Potential of MN, MS, and IMI measured under HCD to predict BW gain under HFD. The correlations have been computed with the 12 rats or after exclusion of the 2 outliers (triangles). 

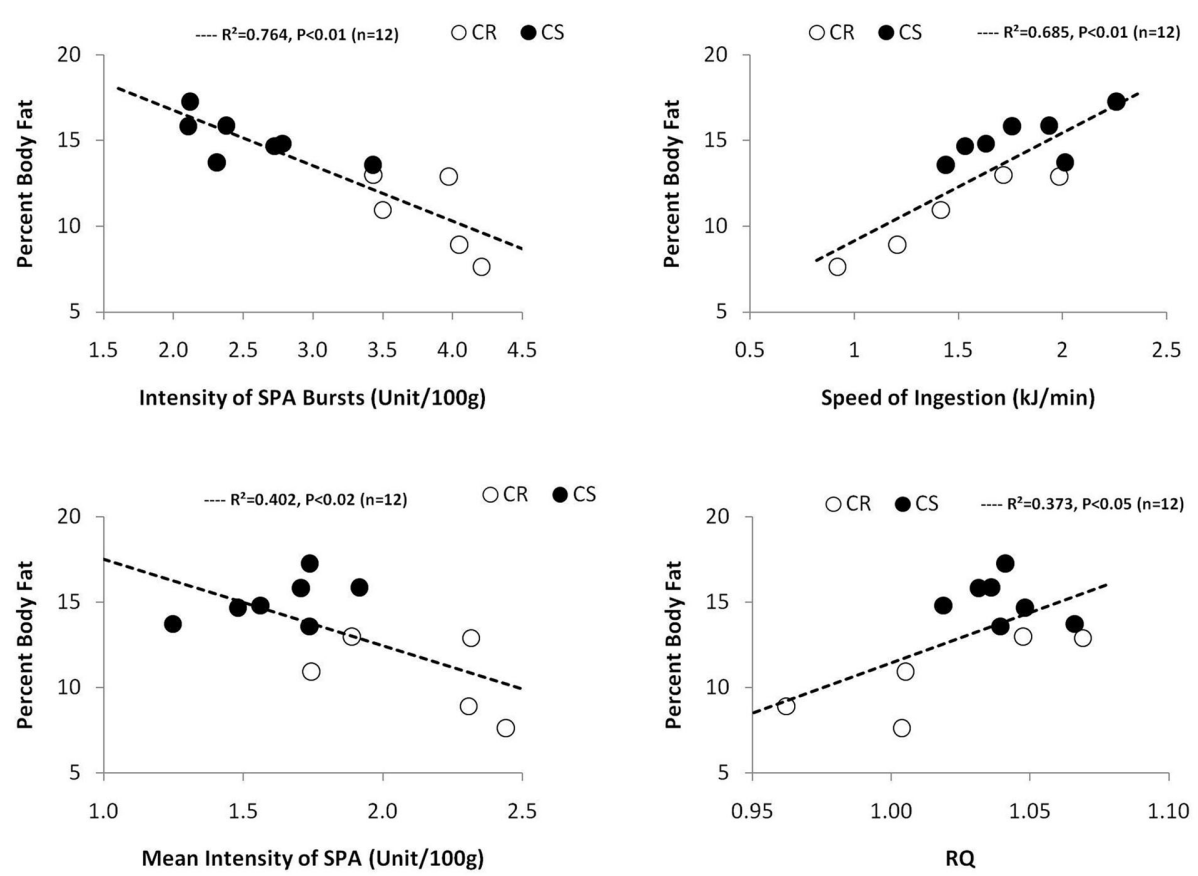

FIGURE 3 | Potential of intensity of SPA bursts, speed of ingestion, and mean intensity of SPA and RO measured under HCD to predict development of body adiposity under HFD (all 12 rats included).

confident that this probably did not affect discrimination between FR and FS rats, the rate of BW gain decreasing only slowly over months. It is more difficult however to judge how this may have affected body composition. Encouragingly, in our hands body adiposity in male Wistar rats as measured by dissection analysis increases by less than 10\% from 200 to $390 \mathrm{~g}$ (Even et al., 2001).

With respect to the CR/CS separation, this assumes that between-group differences in body fat accumulated during the 6-day exposure to HFD is a minor component of that accumulated over the entire study period. More generally, the grouping of the rats into CR/CS and FR/FS is limited by the lack of longitudinal monitoring of adipose mass throughout the various phases of this study. We intend to rectify this situation in the future by using MRI.

It should also be mentioned that the relatively short exposure of 6 days to HFD may be a limiting factor in discriminating between the FS and FR phenotypes. We would like to argue however that in most studies published hitherto FR and FS rats were separated from their BW gain and that much respected research groups (Pagliassotti et al., 1993; Commerford et al., 2001) have already shown that a 6-day period is long enough to discriminate between FR and FS subjects. On the other hand, the validity of the selection of the CR and CS rats after they were submitted to 6 days of HFD is indeed more disputable. We are, however, strongly convinced that the 6-day period of HFD did not significantly alter differentially the adiposity level of the CR and CS rats because the BW gain of these two groups during the HFD period was very similar (27.4 vs. $25.7 \mathrm{~g}$ ). Also, as quoted above, the fundamentals of the selection between FR and FS rats used by most laboratories is that during HFD BW gain and body fat gain are highly correlated. In addition, recent unpublished MRI data obtained in our group indeed show that in our hands, the $R^{2}$ between body fat and BW gain during HFD is 0.767 .

The goal of this study was to distinguish rats according to their sensitivity to HFD (FS vs. FR), but unexpected differences in body adiposity observed at the end of the study led us to also discriminate $\mathrm{CR}$ and $\mathrm{CR}$ rats and study sensitivity to HCD. We analyzed if differences in feeding, SPA and various components of energy metabolism could be predictive for these sensitivities to the diets. We observed that FS and CS rats exhibited different and specific metabolic and behavioral characteristics. Some of these characteristics were potentially reliable and non-invasive predictors to rapidly differentiate FS and CS rats while still lean and fed a usual HCD.

\section{CHARACTERIZATION OF FS AND FR RATS}

It is noteworthy that the FS rats did not gain more weight (therefore most probably not more fat) than the FR groups under HCD. Diets high in fat and consequently of higher energy density encourage food consumption (Poppitt, 1995), and accordingly in this study CI was larger during HFD than during HCD for all rats. However, FS rats did not eat significantly more food (g) than FR rats under either HFD or HCD. In contrast, FS rats ate significantly more meals under both diets but had smaller meals during the two first days of HFD. However, the reduction in meal size was only transient and vanished after 2-3 days of HFD. The fact that meal size was not larger in FS rats tends to contradict the idea that FS rats eat larger meals. However, such observations are usually made on rats that are already overweight after high fat feeding (Farley et al., 2003) which may confound pre-existing differences 
in meal pattern with current obesity, metabolic, and endocrine adaptation to HFD.

That FS rats eat more meals than FR rats when fed a HCD has been reported previously in genetically selected FR and FS rats (Ricci and Levin, 2003; Cottone et al., 2007). Interestingly, these genetically selected rats seem similar to the outbred rats of this study since they were as lean as or even leaner than their FR counterparts when maintained under HCD. Meal fractioning has been proposed as a means to oppose obesity in humans (Cohn et al., 1965; Nicklas et al., 2001; Parks and McCrory, 2005) which contradicts the present observation that meal fractioning is associated with an increased sensitivity to obesity. In fact, meal fractioning does not seem to influence the predisposition to gain weight or fat. In FS rats, meal fractioning was higher under HCD when FS rats did not gain more weight or fat than FR rats, and was maintained under HFD when FS rats gained more weight. Therefore, meal fractioning is probably not responsible per se for the higher sensitivity to HF feeding in FS rats. Rather it may reveal some defect in the mechanisms controlling FI. For example, among potential defective signal(s), leptin production, or central sensitivity to leptin may be postulated. Indeed, central administration of leptin has been shown to reduce meal frequency in rats (Zorrilla et al., 2005) and to favor post-meal satiety (Montague et al., 1997; Chapelot et al., 2000; WesterterpPlantenga et al., 2001). A defect in leptin sensitivity has also been reported in genetically selected FS rats (Levin et al., 2004; Clegg et al., 2005; Irani et al., 2007). Thus, measurement of meal number under HCD seems to be a criterion to easily and noninvasively separate FR and FS rats in a colony of young, non-obese unselected outbred rats, bearing in mind however that while there is indeed a good correlation, there is also overlap of individual values.

The fact that hyperphagia is a characteristic of FS rats is widely accepted and was even suggested to be a faithful predictor of sensitivity to obesity (Dourmashkin et al., 2006). Hyperphagia as the critical stimulus to increase BW gain in FS rats fed a HFD has also been suggested by the observation that FS rats have no increased inherent capacity for dietary fat retention (Commerford et al., 2001). However, in virtually every animal model of obesity in which hyperphagia is a characteristic feature, increased fat deposition has been shown to be independent of the increase in FI (Friedman, 1990, 1998). Therefore, if hyperphagia cannot be ruled out as a component of the sensitivity to HFD, at least for some of the FS rats, the present results confirm that increased FI is only one among several components responsible for increased weight gain of FS subjects under HFD.

Spontaneous physical activity is considered an important component involved in the resistance to HFD (Levine et al., 1999), but analysis of the various components of activity in FS and FR rats in this study did not reveal any significant difference, neither during HCD nor during HFD. In line with this absence of differences, genetically selected FS rats have not been shown to be less active than FR rats under HCD but to become less active only after overweight develops following long-term HFD (29 days; Novak et al., 2006). Thus, following the lack of a short-term effect of the HFD on SPA observed here, it is probable that the decrease in SPA after long-term HFD accompanies or results from, but is not responsible for, the gain in weight and the development of metabolic disturbances that progress during HFD.

Analysis of TEE and RQ suggests that the multiple pre-existing abnormalities reported in FS rats do not significantly affect these components of energy expenditure under HCD, a result that can be considered as normal since FS and FR rats maintained under the HCD ended the study with the same BW, LBM, carcass mass, fat mass, and adiposity. In contrast, during transition to the HF diet, TEE decreased more in FS rats while RQ tended to decrease less so that after 3 days of HFD RQ was higher in FS rats. These responses are obviously able to promote fat storage under HFD by saving energy and reducing fat oxidation. Therefore, studying the transition from HCD to HFD was able to reveal metabolic defects that were not visible under HCD. This suggests that processes specifically related to partitioning of dietary lipids between storage and oxidation are affected in FS rats during HFD but not HCD. Many potential mechanisms have been suggested in the literature, but one possible mechanism that we consider worth further investigation may be a defective lipid oxidation located specifically in muscles which progressively leads to fat accumulation in muscle and insulin resistance if HFD is continued (Galgani et al., 2008). To our knowledge, there has been no direct demonstration that this phenomenon pre-exists in still lean FS rats, but it has been reported that a significantly lower proportion of type I muscle fibers in the medial head of the gastrocnemius muscle of FS rats already before HFD may play a role in determining susceptibility to dietary obesity (Abou Mrad et al., 1992). Thus, changes in TEE and RQ during the first days of adaptation to HFD are potential parameters to discriminate between FR and FS rats.

\section{CHARACTERIZATION OF CR AND CS RATS}

Classification of CR and CS rats according to their body fat content at the end of the study showed that despite large differences in body fat, CS rats were only slightly, not significantly heavier than the CR ones, and had similar LBM and carcass mass. This may explain why this kind of rat had not been clearly distinguished, except, to our knowledge, in one publication (Dourmashkin et al., 2005) in which overfeeding and BW gain was boosted by a high content of saccharose in the diet which was not the case here. In addition we did not find in this study (but the number of rats was small) that CS rats were more sensitive to HFD than CR ones.

Contrary to what was observed between FR and FS rats, no differences were observed between CR and CS rats in any of the components of meal pattern under either HCD or HFD. In contrast, differences were observed at the level of SPA. CR rats developed bursts of SPA of higher intensity than the CS ones. The difference was particularly strong and significant during HCD, which is when the rats were fed the diet for which they exhibited a different sensitivity for fat deposition. The bursts of SPA correspond to the intensity of the mechanical work developed when the rats are active in contrast to the mean SPA that is the mean mechanical work developed during $24 \mathrm{~h}$ which includes resting periods. This component cannot be quantified with the usual redbeams device but requires a method able to quantitatively measure the forces that develop on the floor of the cage by force transducers located beneath the cage. We have extensive experience with this system and have been able to control that the intensity of the 
activity signal tightly correlates to the energy expended in relation to muscular work (Even et al., 1994).

In humans, a reduced SPA is considered a trait of individuals sensitive to obesity and cardiovascular problems under HFD (Moore, 2000) and the increasingly sedentary habits associated with modern lifestyles are suggested to play an important role in the development of obesity (Prentice and Jebb, 1995). However, the influence on adiposity of the type of activity in which people engage during their daily life, or the way they perform standardized activities (walking, stair-climbing, has not been systematically investigated. Here, we observed that the intensity of the bursts of SPA was very significantly lower in CS rats during HCD. This result suggests that rather than, or in conjunction with the absolute amount of activity, the type of activity, and specifically its intensity or its briskness may be involved in the resistance/sensitivity to fat deposition under $\mathrm{HCD}$, possibly by influencing the control of energy metabolism and the aerobic/glycolytic capacities in muscles. For example, it was recently shown that rats with greater aerobic capacities were more active and also more resistant to HFD-induced obesity and responded differently to i.c.v. injections of orexin-A (Novak et al., 2010). Thus, activity and aerobic capacities in muscle are both important components of the response to HFD and we bring here preliminary data suggesting that this may well also be the case in rats that exhibit different sensitivities to HCD. On practical grounds, the amplitude of the differences in the bursts of activity between CS and CR rats makes this component of activity a potentially robust parameter to separate the two groups. It is in fact the only parameter for which we observed no overlap between CR and CS rats. This observation deserves further attention to confirm this phenomenon and further analyze how it is connected with the increased adiposity that develops in CS rats, in particular to what extent muscle metabolism is involved.

Measurements of TEE did not reveal differences between CR and CS rats. Comparison of CI with TEE showed that energy balance (EB) was not significantly different in the two groups under $\mathrm{HC}$ as well as under HF feeding. The increase in EB during HF feeding was mainly the result of an increase in CI while EE was unchanged, the amplitude of this phenomenon being comparable in the two groups. The fact that TEE does not increase (or only marginally so) after HF feeding has been reported in various studies (Dallosso and James, 1984; Schutz et al., 1989). However, it is interesting to note that despite not being significantly different, EB was steadily higher in CS than in CR rats. The difference was the largest $(25 \%)$ under HC feeding. This was confirmed by differences between RQ values that were also larger in CS rats. The fact the EB and RQ values were not significantly different may be disappointing, but one must keep in mind that obesity develops

\section{REFERENCES}

Abou Mrad, J., Yakubu, F., Lin, D., Peters, J. C., Atkinson, J. B., and Hill, J. O. (1992). Skeletal muscle composition in dietary obesity-susceptible and dietary obesity-resistant rats. Am. J. Physiol. 262, R684-R688.

Chang, S., Graham, B., Yakubu, F., Lin, D., Peters, J. C., and Hill, J. O. (1990). Metabolic differences between obesity-prone and obesityresistant rats. Am. J. Physiol. 259, R1103-R1110.

Chapelot, D., Aubert, R., Marmonier, C., Chabert, M., and Louis-Sylvestre, J. (2000). An endocrine and metabolic definition of the intermeal interval in humans: evidence for a role of leptin on the prandial pattern through fatty acid

very progressively as a result a very tiny daily differences that are necessarily very difficult to reveal by direct measurement of energy expenditure. Differences in RQ have also been reported under HF feeding between FS and FR (Chang et al., 1990), and the authors suggested that the capacity to adjust nutrient oxidation to nutrient intake was a major mechanism underlying the sensitivity to HF feeding. This is obviously a potential mechanism, and it seems logical that in the CS rats of the present study, differences in RQ may be larger under HCD than under HFD.

\section{CONCLUSION}

The study reported here was devoted to revealing parameters giving early indications of a potential sensitivity to increased adiposity under HFD and show that rats differ not only in their sensitivity to HFD but also in their sensitivity to HCD, this latter sensitivity being more difficult to reveal because it induces only small differences in BW gain.

One major point to emphasize is that we did not eliminate any individuals from the group of 12 rats that were introduced in the study, but rather asked the breeder to provide us heterogeneous rats from their colony. Despite the initial disappointing observation that the difference in BW between the light and heavy rats was small, this approach probably helped to discriminate FS and FR rats as well as CR and CS rats within a small group and to reveal significant correlations between various metabolic and behavioral parameters and the level of sensitivity to HFD as well as HCD.

The best predictors of the dietary sensitivity of the rats are behavioral parameters related to feeding behavior, mainly SPA. RQ is also a potential predictor, but the greater difficulty to get a precise and reproducible measure together with the overlap of individual values makes RQ useful only for discrimination between extreme individuals.

\section{ACKNOWLEDGMENTS}

This study was funded by grants from the Institut national de la rechercheagronomique (French National Institute for Agricultural Research, INRA), Centre National de la Recherche Scientifique (French National Center for Scientific Research, CNRS), and the "Institut Benjamin Delessert." This work was primarily that of Patrick Christian Even, who was the main instigator at all stages. Gilles Fromentin and Daniel Tomé helped conceive the work, Catherine Chaumontet and Dalila Azzout aided in body composition measurement, Nachiket A. Nadkarni assisted in data analysis, Nachiket A. Nadkarni and Daniel Tomé contributed to writing this manuscript. The authors would like to thank AngéliqueSimonin and JulienPiedcoq for their help in the management of the rats and their participation in carcass analyses.

disposal. Am. J. Clin. Nutr. 72, 421-431.

Clegg, D. J., Benoit, S. C., Reed, J. A., Woods, S. C., Dunn-Meynell, A., and Levin, B. E. (2005). Reduced anorexic effects of insulin in obesity-prone rats fed a moderate-fat diet. Am. J. Physiol. Regul. Integr. Comp. Physiol. 288, R981-R986.
Cohn, C., Joseph, D., Bell, L., and Allweiss, M. D. (1965). Studies on the effects of feeding frequency and dietary composition on fat deposition. Ann. N. Y. Acad. Sci. 131, 507-518.

Commerford, S. R., Pagliassotti, M. J., Melby, C. L., Wei, Y., and Hill, J. O. (2001). Inherent capacity for lipogenesis or dietary fat retention is not 
increased in obesity-prone rats. Am. J. Physiol. Regul. Integr. Comp. Physiol. 280, R1680-R1687.

Cottone, P., Sabino, V., Nagy, T. R., Coscina, D. V., and Zorrilla, E. P. (2007). Feeding microstructure in diet-induced obesity susceptible versus resistant rats: central effects of urocortin 2. J. Physiol. (Lond.) 583, 487-504.

Dallosso, H. M., and James, W. P. (1984). Whole-body calorimetry studies in adult men. 1. The effect of fat overfeeding on 24 h energy expenditure. Br. J. Nutr. 52, 49-64.

Dourmashkin, J. T., Chang, G. Q., Gayles, E. C., Hill, J. O., Fried, S. K., Julien, C., and Leibowitz, S. F. (2005). Different forms of obesity as a function of diet composition. Int. J. Obes. (Lond.) 29, 1368-1378.

Dourmashkin, J. T., Chang, G. Q., Hill, J. O., Gayles, E. C., Fried, S. K., and Leibowitz, S. F. (2006). Model for predicting and phenotyping at normal weight the long-term propensity for obesity in SpragueDawley rats. Physiol. Behav. 87, 666-678.

Even, P. C., Mokhtarian, A., and Pele, A. (1994). Practical aspects of indirect calorimetry in laboratory animals. Neurosci. Biobehav. Rev. 18, 435-447.

Even, P. C., Rolland, V., Roseau, S., Bouthegourd, J. C., and Tome, D. (2001). Prediction of basal metabolism from organ size in the rat: relationship to strain, feeding, age, and obesity. Am. J. Physiol. Regul. Integr. Comp. Physiol. 280, R1887-R1896.

Farley, C., Cook, J. A., Spar, B. D., Austin, T. M., and Kowalski, T. J. (2003). Meal pattern analysis of diet-induced obesity in susceptible and resistant rats. Obes. Res. 11, 845-851.

Ferrannini, E. (1988). The theoretical bases of indirect calorimetry: a review. Metab. Clin. Exp. 37, 287-301.

Flatt, J. P. (1987). The difference in the storage capacities for carbohydrate and for fat, and its implications in the regulation of body weight. Ann. N. Y. Acad. Sci. 499, 104-123.

Flatt, J. P. (1988). Importance of nutrient balance in body weight regulation. Diabetes Metab. Rev. 4, 571-581.

Friedman, M. I. (1990). Body fat and the metabolic control of food intake.
Int. J. Obes. 14(Suppl. 3), 53-66; discussion 66-57.

Friedman, M. I. (1998). Fuel partitioning and food intake. Am. J. Clin. Nutr. 67, 513S-518S.

Galgani, J. E., Moro, C., and Ravussin, E. (2008). Metabolic flexibility and insulin resistance. Am. J. Physiol. Endocrinol. Metab. 295, E1009-E1017.

Irani, B. G., Dunn-Meynell, A. A., and Levin, B. E. (2007). Altered hypothalamic leptin, insulin, and melanocortin binding associated with moderate-fat diet and predisposition to obesity. Endocrinology 148, 310-316.

Ji, H., and Friedman, M. I. (2003). Fasting plasma triglyceride levels and fat oxidation predict dietary obesity in rats. Physiol. Behav. 78, 767-772.

Lacroix, M., Gaudichon, C., Martin, A., Morens, C., Mathe, V., Tome, D., and Huneau, J. F. (2004). A long-term high-protein diet markedly reduces adipose tissue without major side effects in Wistar male rats. Am. J. Physiol. Regul. Integr. Comp. Physiol. 287, R934-R942.

Layman, D. K., Evans, E. M., Erickson, D., Seyler, J., Weber, J., Bagshaw, D., Griel, A., Psota, T., and KrisEtherton, P. (2009). A moderateprotein diet produces sustained weight loss and long-term changes in body composition and blood lipids in obese adults. J. Nutr. 139, 514-521.

Levin, B. E., Dunn-Meynell, A. A., Balkan, B., and Keesey, R. E. (1997). Selective breeding for diet-induced obesity and resistance in SpragueDawley rats. Am. J. Physiol. 273, R725-R730.

Levin, B. E., Dunn-Meynell, A. A., and Banks, W. A. (2004). Obesity-prone rats have normal blood-brain barrier transport but defective central leptinsignaling before obesity onset. Am. J. Physiol. Regul. Integr. Comp. Physiol. 286, R143-R150.

Levine, J. A., Eberhardt, N. L., and Jensen, M. D. (1999). Role of nonexercise activity thermogenesis in resistance to fat gain in humans. Science 283, 212-214.

Montague, C. T., Farooqi, I. S., Whitehead, J. P., Soos, M. A., Rau, H., Wareham, N. J., Sewter, C. P., Digby, J. E., Mohammed, S. N., Hurst, J. A. Cheetham, C. H., Earley, A. R., Barnett, A. H., Prins, J. B., and O’Rahilly,
S. (1997). Congenital leptin deficiency is associated with severe earlyonset obesity in humans. Nature 387, 903-908.

Moore, M. S. (2000). Interactions between physical activity and diet in the regulation of body weight. Proc. Nutr. Soc. 59, 193-198.

Neel, J. V. (1999). The "thrifty genotype" in 1998. Nutr. Rev. 57, S2-S9.

Nicklas, T. A., Baranowski, T., Cullen, K. W., and Berenson, G. (2001). Eating patterns, dietary quality and obesity. J. Am. Coll. Nutr. 20, 599-608.

Novak, C. M., Escande, C., Burghardt, P. R., Zhang, M., Barbosa, M. T., Chini, E. N., Britton, S. L., Koch, L. G., Akil H., and Levine, J. A. (2010). Spontaneous activity, economy of activity, and resistance to diet-induced obesity in rats bred for high intrinsic aerobic capacity. Horm. Behav. 58, 355-367.

Novak, C. M., Kotz, C. M., and Levine, J. A. (2006). Central orexin sensitivity, physical activity, and obesity in dietinduced obese and diet-resistant rats. Am. J. Physiol. Endocrinol. Metab. 290, E396-E403.

Pagliassotti, M. J., Shahrokhi, K. A., and Hill, J. O. (1993). Skeletal muscle glucose metabolism in obesityprone and obesity-resistant rats. Am. J. Physiol. 264, R1224-R1228.

Parks, E. J., and McCrory, M. A. (2005). When to eat and how often? Am. J. Clin. Nutr. 81, 3-4.

Pichon, L., Huneau, J. F., Fromentin, G., and Tome, D. (2006). A highprotein, high-fat, carbohydrate-free diet reduces energy intake, hepatic lipogenesis, and adiposity in rats. $J$. Nutr. 136, 1256-1260

Poppitt, S. D. (1995). Energy density of diets and obesity. Int. J. Obes. Relat. Metab. Disord. 19(Suppl. 5), S20-S26.

Prentice, A. M., Hennig, B. J., and Fulford, A. J. (2008). Evolutionary origins of the obesity epidemic: natural selection of thrifty genes or genetic drift following predation release? Int. J. Obes. (Lond.) 32, 1607-1610.

Prentice, A. M., and Jebb, S. A. (1995). Obesity in Britain: gluttony or sloth? BMJ 311, 437-439.

Ricci, M. R., and Levin, B. E. (2003). Ontogeny of diet-induced obesity in selectively bred Sprague-Dawley rats. Am. J. Physiol. Regul. Integr. Comp. Physiol. 285, R610-R618.
Schutz, Y., Flatt, J. P., and Jequier, E. (1989). Failure of dietary fat intake to promote fat oxidation: a factor favoring the development of obesity. Am. J. Clin. Nutr. 50, 307-314.

Speakman, J. R. (2007). Anonadaptive scenario explaining the genetic predisposition to obesity: the "predation release" hypothesis. Cell Metab. 6, 5-12.

Stoger, R. (2008). The thrifty epigenotype: an acquired and heritable predisposition for obesity and diabetes? Bioessays 30, 156-166.

Westerterp-Plantenga, M. S., Saris, W. H., Hukshorn, C. J., and Campfield, L. A. (2001). Effects of weekly administration of pegylated recombinant human $\mathrm{OB}$ protein on appetite profile and energy metabolism in obese men. Am. J. Clin. Nutr. 74, 426-434.

Zorrilla, E. P., Inoue, K., Valdez, G. R., Tabarin, A., and Koob, G. F. (2005). Leptin and post-prandial satiety: acute central leptin more potently reduces meal frequency than meal size in the rat. Psychopharmacology (Berl.) 177, 324-335.

Conflict of Interest Statement: The authors declare that the research was conducted in the absence of any commercial or financial relationships that could be construed as a potential conflict of interest.

Received: 21 September 2011; accepted: 22 November 2011; published online: 16 December 2011.

Citation: Even PC, Nadkarni NA, Chaumontet C, Azzout-Marniche D, Fromentin $G$ and Tomé D (2011) Identification of behavioral and metabolic factors predicting adiposity sensitivity to both high fat and high carbohydrate diets in rats. Front. Physio. 2:96. doi: 10.3389/fphys.2011.00096

This article was submitted to Frontiers in Integrative Physiology, a specialty of Frontiers in Physiology.

Copyright (c) 2011 Even, Nadkarni, Chaumontet, Azzout-Marniche, Fromentin and Tomé. This is an open-access article distributed under the terms of the Creative Commons Attribution Non Commercial License, which permits noncommercial use, distribution, and reproduction in other forums, provided the original authors and source are credited. 